\title{
Accuracy of Breath-hold CT in Treatment Planning for Lung Stereotactic Ablative Radiotherapy
}

Dominique Mathieu ${ }^{1}$, Charles Martel ${ }^{2}$, Marie-Pierre Campeau ${ }^{1}$, Édith Filion ${ }^{2}$, David Roberge $^{3}$, Houda Bahig ${ }^{2}$, Toni Vu ${ }^{2}$, Louise Lambert ${ }^{2}$, Karim Boudam ${ }^{4}$, Jean-Francois Carrier $^{2}$

1. Department of Radiation Oncology, Centre hospitalier de l'Université de Montréal (CHUM) 2. Department of Radiation Oncology, Centre hospitalier de l'Université de Montréal (CHUM) 3. Department of Oncology, Division of Radiation Oncology, McGill University Health Center 4. Department of Radiation Oncology, Centre hospitalier de l'Université de Montréal (CHUM)

$\square$ Corresponding author: Dominique Mathieu, mathieu_dominique1@hotmail.com Disclosures can be found in Additional Information at the end of the article

\section{Abstract}

Purpose: The objectives of this study are (1) to measure concordance of tumor position on breath-hold (BH) computed tomography (CT) scans relative to the natural tumor path during free breathing (FB) and (2) to evaluate the benefits of the breathing monitoring device Abches (Apex Medical, Tokyo) for stereotactic ablative radiotherapy (SABR) treatment planning.

Methods: In 53 lung cancer patients treated with CyberKnife ${ }^{\mathrm{TM}}$ robotic radiosurgery system, FB four-dimensional computerized tomography (4DCT) and end-expiration (EE) BH CT images were obtained. Extent of natural tumor motion was assessed with rigid registration derived from end-inspiration (EI) and EE phases of the 4DCT. Tumor displacement in BH scans relative to the natural tumor path was measured relative to the EE 4DCT phase.

Results: Mean tumor motion (+/- $1 \mathrm{SD})$ during natural FB was $1 \pm 1 \mathrm{~mm}, 2 \pm 2 \mathrm{~mm}$, and $6 \pm 6 \mathrm{~mm}$ in medio-lateral, anterior-posterior, and cranio-caudal directions, respectively. Tumor position on BH CT scan was closer to EE than EI 4DCT phase for 35/53 patients (66\%). Difference of BH tumor position vs. EE state was $4 \pm 3 \mathrm{~mm}$. Gross tumor displacements perpendicular to natural tumor path were as great as $11 \mathrm{~mm}$ (anterior-posterior) and were seen with or without the breathing monitoring device.

Conclusion: Tumor position during BH CT may not accurately correspond to positions observed on FB 4DCT. Hence, accurate and custom 4D analysis for each individual patient is

Received 11/27/2014

Review began 11/27/2014

Review ended 12/21/2014

Published 12/22/2014

@ Copyright 2014

Mathieu et al. This is an open access article distributed under the terms of the Creative Commons Attribution License CC-BY 3.0., which permits unrestricted use, distribution, and reproduction in any medium, provided the original author and source are credited.
Categories: Radiation Oncology

Keywords: sabr, cyberknife ${ }^{\mathrm{TM}}$, treatment planning, breath-hold, abches, 4dct

\section{Introduction}

Stereotactic ablative radiotherapy (SABR) is the standard treatment for medically inoperable patients with early stage non-small cell lung cancer (NSCLC). This technique delivers highly focused doses ranging from 50-60 Gy in three to five fractions, depending on tumor location and dose constraints to organs at risk (OAR). At Centre Hospitalier de l'Université de Montréal 


\section{Cureus}

(CHUM), CyberKnife ${ }^{\mathrm{TM}}$ (Accuray Inc., Sunnyvale, CA) SABR treatment planning includes a fourdimensional computed tomography (4DCT) scan during free breathing (FB) and a breath-hold (BH) CT scan used for dose calculation.

The aim of this study is to measure conformity of tumor position on BH CT scans relative to natural tumor path during free breathing $(\mathrm{FB})$ and to evaluate a potential benefit of the Abches device (Apex Medical, Tokyo, Japan) in BH CT scanning. Abches is a breathing monitoring device that allows the patient to self-control the respiratory motion of the chest and abdomen [1].

\section{Materials And Methods}

\section{Patients and treatment}

Fifty-three early stage NSCLC patients were included in this retrospective study. Patients underwent SABR with the CyberKnife ${ }^{\mathrm{TM}}(\mathrm{CK})$ robotic radiosurgery system using near-real time tumor tracking or an internal target volume (ITV) technique. Informed consent was obtained by all participants in this study. Patients and treatment characteristics are summarized in Table 1. 


\section{Cureus}

\section{Sex}

Male

$29(55 \%)$

Female

$24(45 \%)$

Median age (range)

$76(44-87)$

\section{T-classification}

T1a

$26(49 \%)$

T1b

$14(26 \%)$

T2a

$12(23 \%)$

T3

$1(2 \%)$

\section{Tumor location}

Upper lobes

Lower and middle lobes

Left lung

Right lung

$30(57 \%)$

\section{Tracking technique}

Fiducials

Direct soft tissue

Spine-based setup

$20(38 \%)$

\section{BH CT acquisition}

No monitoring device

\section{TABLE 1: Patient and treatment characteristics $(n=53)$}

Tumor tracking was either performed with a fiducial approach after a placement of radioopaque markers or with direct soft tissue tracking (Xsight ${ }^{\circledR}$ Lung) for patients with lesions trackable on stereoscopic images [2]. Gross tumor volume (GTV) delineation was performed in a lung window setting on the BH CT scan.

Xsight ${ }^{\circledR}$ Spine Tracking was used for patients with tumors located close to the spine with minimal breathing movement. Internal gross tumor volume (IGTV) contours were obtained from the BH planning CT and the extreme respiratory phases of the 4DCT. For tumors exhibiting hysteresis in their movement, IGTV also contained any relevant 4DCT phase. 
Planning CT images were obtained with the Brilliance CT Big Bore (Philips Medical Systems, Cleveland, $\mathrm{OH}$ ) and included a FB 4DCT scan as well as a BH CT scan at end expiration (EE). Both scans were acquired without intravenous contrast in supine position with a custom foam cushion as the only immobilization device.

The purpose of the 4DCT was to (1) evaluate natural tumor motion, (2) locate radio-opaque markers to be used for tracking, and (3) point out tumor presenting significant deformation or rotation during the respiratory cycle. A 4DCT scan was targeted on the lesion with $\pm 6 \mathrm{~cm}$ added in the superior-inferior direction. Two millimeter thick CT slices were acquired every $1 \mathrm{~mm}$. During acquisition, patients were instructed to breathe normally. No audio coaching was used. Patient breathing pattern was obtained with the Bellows system ${ }^{\circledR}$ (Philips Medical Systems, Cleveland, OH). Prior to imaging, the simulation team ensured that the respiratory rate was 10 20 breaths per minute and stable in amplitude. A phase-based sorting method was used to reconstruct CT images into three-dimensional (3D) bins at 10 different respiratory phases.

The BH CT scan was used to conduct treatment planning on MultiPlan, the CK planning software (version 4.5, Accuray Inc., Sunnyvale, CA). This acquisition mode reduces motion artifacts and improves the quality of digitally reconstructed radiographs used for CK tracking. $\mathrm{BH}$ during EE state was preferred since the exhalation phase is dominant in the normal respiratory cycle and typically better represents the average tumor position [3]. In an ideal $\mathrm{BH}$ planning CT scan, the tumour should sit within its natural FB trajectory, preferably as close as possible to its EE position. The BH CT scan imaged the patients from adrenals to chin and acquired $2 \mathrm{~mm}$ slices every $1 \mathrm{~mm}$. The usual acquisition time was approximately 10 seconds. As part of the $\mathrm{BH}$ acquisition without the Abches device, the respiratory trace from the Bellows system ${ }^{\circledR}$ was monitored and the patient was instructed to stop breathing in EE. Patient rehearsals insured that end-expiratory $\mathrm{BH}$ could be performed at the time of acquisition.

\section{Breathing control device}

The Abches breathing monitoring device has been described in previous publications [1, 4-7]. The device allows patients to self-monitor their breathing during imaging and/or treatment. It measures the breathing amplitude using the combined movement of one or two arms positioned on the abdomen and thorax. The movement is transmitted mechanically to a dial that the patient can visualise by means of a mirror attached to his forehead. This dial has three adjustable pins which are used to mark end inspiration (yellow), expiration (red) as well as a reference level for BH (blue). The dial is also visible to CT operators via a camera system.

For 12 patients, the Abches device was used in BH CT acquisition. As patients breathed normally, the blue cursor/pin was positioned at the FB EE state. Using this visual feedback, patients were asked to hold their breath as the indicator needle reached the blue cursor. Before CT acquisition, this exercise was practiced until it felt to be reliable. This coaching process typically required 5 to 10 minutes.

\section{Registration technique and analysis}

All tumor registrations were performed with a lung window setting (width 1500 Hounsfield units [HU]; level $-500 \mathrm{HU}$ ) by aligning GTV to GTV between the different phases or scans through translations. These translations were then quantified. Breathing motion was isolated from patient motion by performing a registration of the corresponding vertebrae in bone window setting (width 2000; level $300 \mathrm{HU}$ ) prior to looking at the lung tumor. These manual registrations were performed independently by two experienced physicists to ensure reproducible measures. 


\section{Cureus}

To assess the extent of natural tumor motion, tumors from EI and EE phases of the 4DCT scan were registered. Tumor motion was assumed to be linear as no significant hysteresis was observed when the 4D motion was reviewed in a movie ("cine") loop.

Tumor displacement in $\mathrm{BH}$ scans relative to the natural tumor path was measured with EE 4DCT phase as reference.

Statistics included Fisher's exact test for contingency table analysis and Mann-Whitney U test for cohort comparisons.

\section{Results}

Results of all 53 patients are presented in Table 2. Tumor rigid registrations of EI and EE phases of the 4DCT scans showed mean natural movement amplitude of $7 \mathrm{~mm}$ (3D vector) made up of a $1 \mathrm{~mm}$ medio-lateral, $2 \mathrm{~mm}$ anterior-posterior, $6 \mathrm{~mm}$ cranio-caudal. Tumor motion was greater for lower lobe (LL) tumors with a mean 3D vector of $11 \mathrm{~mm}$ ( $\max =35 \mathrm{~mm}$ ) compared to $5 \mathrm{~mm}(\max =12 \mathrm{~mm})$ for upper lobe (UL) tumors. In 11 cases (20\%), the amplitude of natural motion was greater than $10 \mathrm{~mm}(8 / 18 \mathrm{LL}$ and $3 / 35 \mathrm{UL})$.

\section{Natural tumor motion $(\mathrm{mm})$}

Amplitude (3D)

Medio-lateral

Anterior-posterior

Cranio-caudal

Module $\geq 10 \mathrm{~mm}$

BH tumor position ( $\mathrm{mm})$

Distance to EE state

Closer to EE

Within $3 \mathrm{~mm}$ of FB path

\section{Mean \pm SD (range)}

$7 \pm 4(0-35)$

$1 \pm 1(0-5)$

$2 \pm 1(0-9)$

$6 \pm 5(0-35)$

n (\%)

$11(21 \%)$

Mean \pm SD (range)

$4 \pm 3(0-28)$

n (\%)

$35(66 \%)$

$40(75 \%)$

TABLE 2: Tumor rigid registration results $(n=53)$

In BH scans, 18 patients (33\%) were found to have a tumor positioned closer to FB EI than EE. The mean difference in tumor position between BH and EE states was $4 \mathrm{~mm} \pm 3 \mathrm{~mm}$. In 40 patients $(75 \%)$, the GTV on the $\mathrm{BH}$ scan was within a $3 \mathrm{~mm}$ radius of tumor trajectory observed on 4DCT. Displacements of the GTV perpendicular to the natural tumor path $\left(\mathrm{d}_{\perp}\right)$ were as great as $11 \mathrm{~mm}$. These issues are illustrated in Figure 1. 


\section{Cureus}

(a)
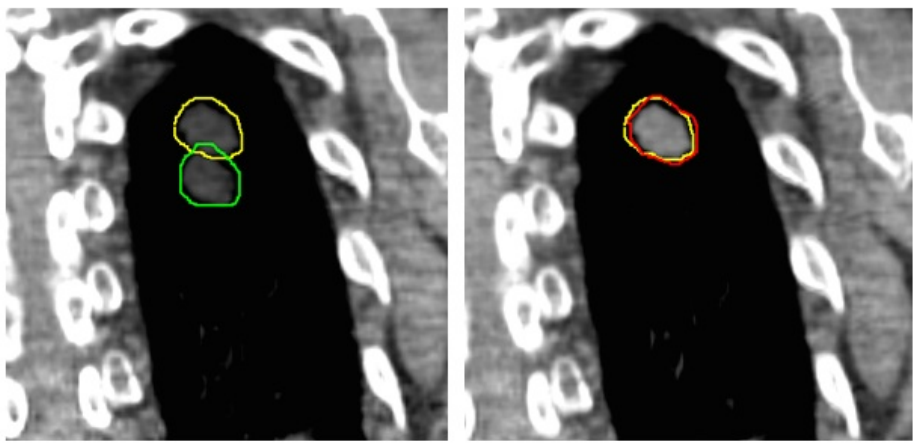

(b)
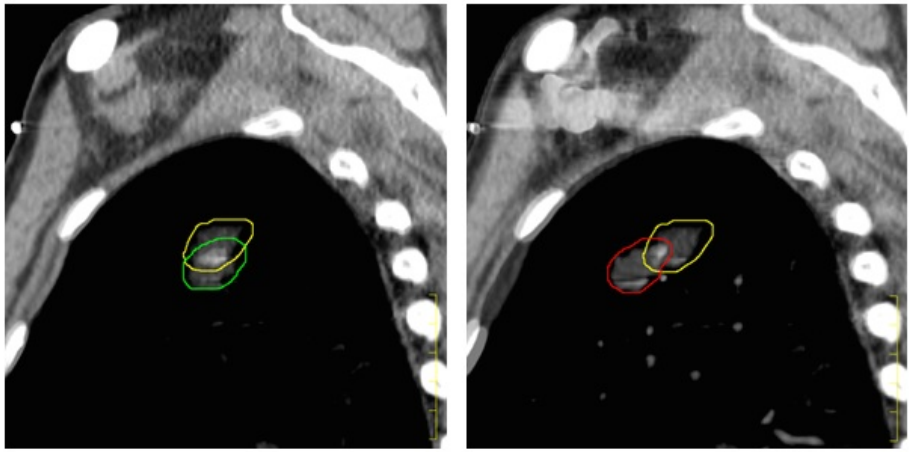

(c)
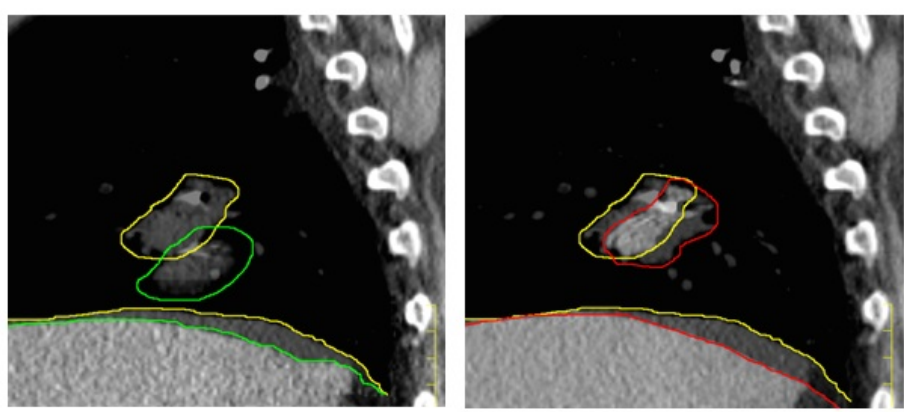

FIGURE 1: Left, CT/CT images fusion of EI (green) and EE (yellow) 4DCT phases representing natural tumor path. Right, CT/CT images fusion of EE 4DCT phase and BH (red) CT.

(a) Ideal BH planning CT acquisition with strict EE phase match, (b) Marginal BH CT acquisition with $\mathrm{d}^{\perp}=11 \mathrm{~mm}$, (c) Marginal BH CT acquisition with $\mathrm{d}^{\perp}=6 \mathrm{~mm}$. All images are presented in mediastinal window setting (width $400 \mathrm{HU}$; level $20 \mathrm{HU}$ ).

Tumor position on BH CT scan was closer to EE than EI 4DCT phase for nine of the 12 patients (75\%) scanned with Abches. Among the 41 patients scanned without a breathing device, the proportion was $63 \%$. This difference did not reach statistical significance (Fisher's exact test $\mathrm{p}=$ $0.8)$.

\section{Discussion}

Over the years, several strategies have been developed to manage respiratory motion during radiotherapy treatments. These include abdominal compression, breath holding, gating, and near real-time tumor tracking. This study addresses tumor position on BH CT scans relative to natural tumor motion and the potential impact of a visual feedback device. Our results suggest that practical difficulties may arise when asking patients to perform a $\mathrm{BH}$ at end-tidal 


\section{Cureus}

expiration. Although tumor position tends to fit within the expected 4DCT trajectory for most patients, distance between BH and FB EE tumor position can be significant as well as unpredictable. This section outlines the potential impacts of inaccurate BH CT scan in treatment planning for CK.

In our patients, natural tumor motion amplitudes ranged from $1 \mathrm{~mm}$ to $35 \mathrm{~mm}$ with a dominant superior-inferior component. Unsurprisingly, tumors located in the LL presented greater motion, as they appear to be predominantly driven by movement of the diaphragm. These results are consistent with other studies assessing 3D tumor motion with different imaging techniques, such as fluoroscopy, slow CT acquisition, 4DCT, and dynamic MRI [8-10]. For ITV based techniques, treatment of tumors with a broad movement enlarges the volume of healthy lungs irradiated and may introduce interplay effect between respiratory motion and beam dynamics [11]. Thus, at our center, these techniques are reserved for tumors with a natural movement less than $10 \mathrm{~mm}$ or that can be decreased to $10 \mathrm{~mm}$ or less with abdominal compression.

Even in those tumors with motion of less than $10 \mathrm{~mm}$, additional healthy lung may be included in an ITV when BH scans are not representative of natural motion. As shown in Figure 2, marginal BH may result in significant increase in treatment volume. In this particular case treated with a spine-based set-up, BH tumor presented a GTV displacement in the anterior direction of $\mathrm{d}_{\perp}=4 \mathrm{~mm}$. Planning target volumes (PTVs) excluding and including BH volumes were 15.7 cc vs $19.6 \mathrm{cc}$, respectively. Wang, et al. have reported similar findings in a study addressing ITV delineation in 10 NSCLC patients treated with SABR [8]. PTV delineated from 4DCT maximum intensity projection (MIP) images resulted in smaller target volumes compared to those obtained with FB 4DCT, EI BH, and EE BH images. The authors had concerns about BH CT acquisition at specific respiratory phases, noting that breath holding could result in aberrant estimation of natural tumor motion.

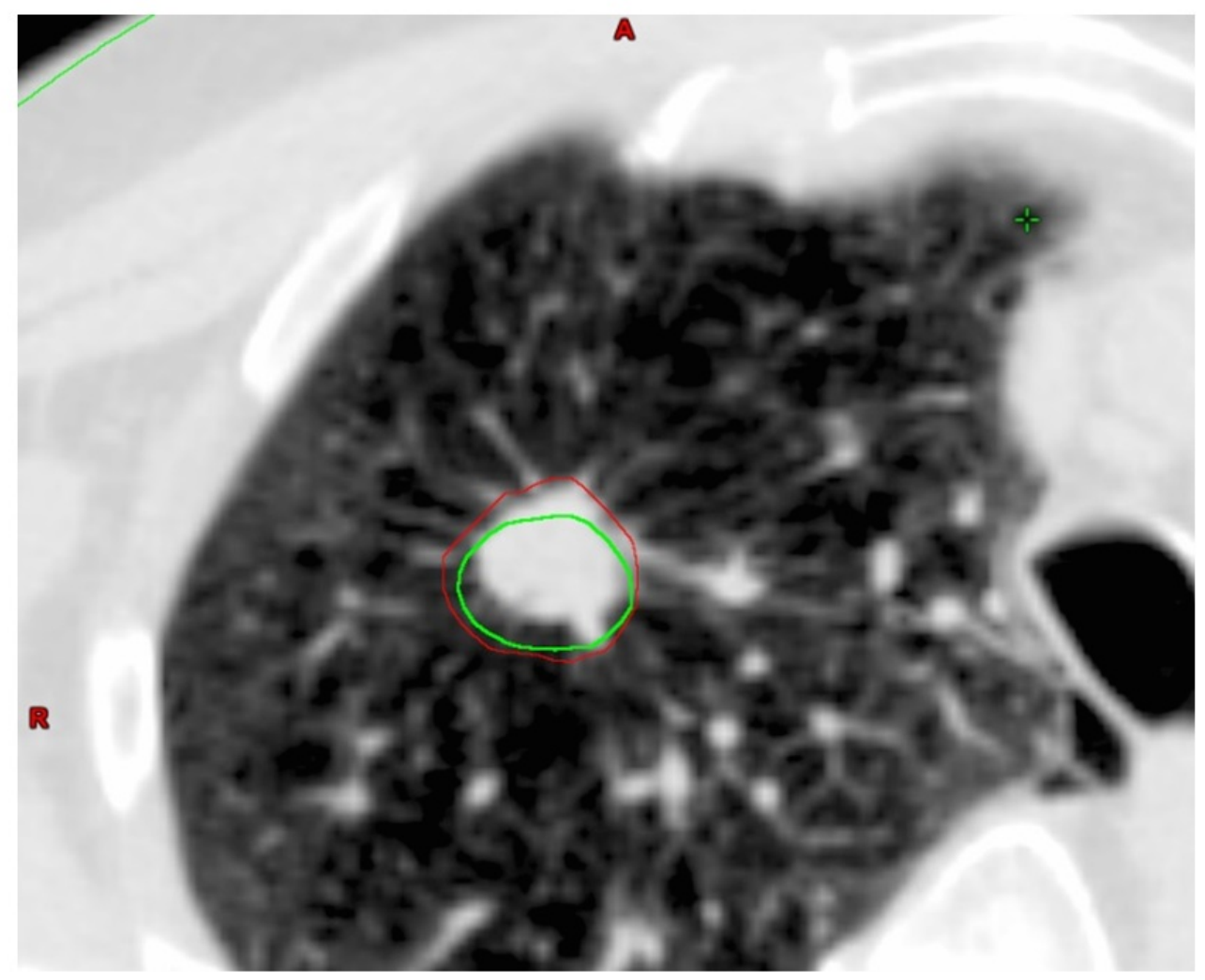

FIGURE 2: CT image of a marginal BH with a GTV 


\title{
Cureus
}

\author{
displacement of $4 \mathrm{~mm}$ in the anterior direction. IGTV contours \\ including (red) and excluding (green) $\mathrm{BH}$.
}

Modes of free breathing treatments, such as robotic tumor tracking, may also be affected by treatment planning conditions. Using marginal BH CT for dose calculation and OARs delineation may result in an underestimation of the effective dose to OARs. Theoretically, this situation can occur if the tumor is more distant from an OAR on a BH scan than it is in FB. This situation is illustrated in Figure 3.

(a)
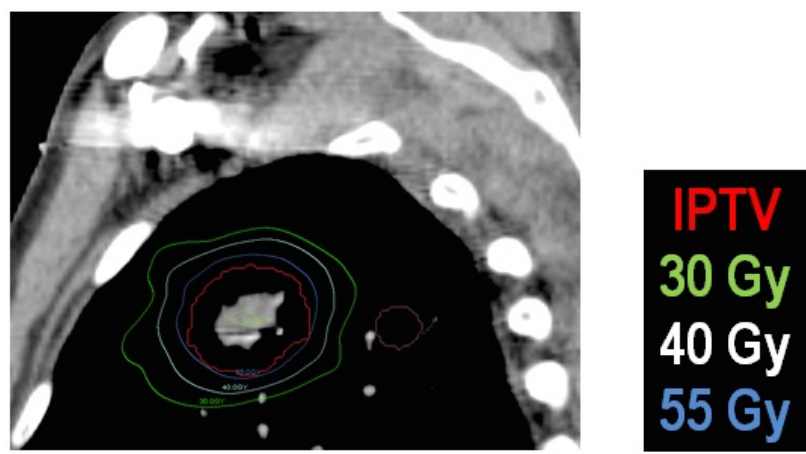

(b)
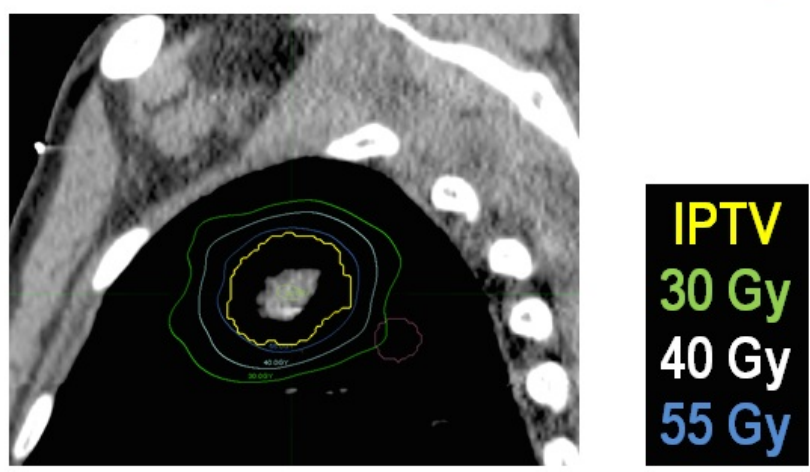

\section{FIGURE 3: CT images and treatment isodoses of a marginal BH with a GTV displacement of $\mathrm{d} \perp=11 \mathrm{~mm}$.}

(a) Isodoses registered on BH GTV, (b) Isodoses registered on EE 4DCT GTV. A fictitious OAR is added in purple to show possible effects. All images are presented in mediastinal window setting (width $400 \mathrm{HU}$; level $20 \mathrm{HU}$ ).

In our study, BH GTV displacements perpendicular to natural tumor path were as great as 11 $\mathrm{mm}$. However, we have not identified any cases for which dosimetric constraints to OARs were impacted. This was confirmed by transposing the dose calculated on BH scans to the EE phase of the corresponding FB 4DCT. Still, we believe it is important to establish a means of detecting unnatural BH scans in the treatment planning process. At our institution, 4DCTs are obtained in all cases of lung SBRT, even when direct tumor tracking will be used. An experienced medical physicist carefully assesses each planning CT scan, and a detailed 4DCT report is produced. This report includes information on natural tumor movement, hysteresis, and BH GTV position relative to EE 4DCT. We believe that this procedure is of value in clinical decision making and can trigger 4D dose calculations when tumor tracking was planned on a BH 3DCT. A sample report is included at the end of the manuscript. 
The benefits of Abches monitoring device to ensure reproducible $\mathrm{BH}$ radiotherapy treatment have been established in several previous studies [1, 4-7]. This device has been extensively used, particularly in Asia, to treat multiple sites, including lung, liver, and breast cancer. In a study including 32 NSCLC, Tarohda, et al. concluded that the Abches device could be effectively used to obtain appropriate reproducibility of tumor position during lung SABR under breath holding conditions [5]. Lee, et al. observed that Abches could be used to treat left-sided breast cancer in a deep inspiration breath-hold setting with potential dose reduction to the heart [7].

However, studies investigating the use of Abches for lung treatment planning are sparse. In this report, we have not identified a statistically significant improvement in BH conformity to FB path using the Abches device. However, our analysis is limited by the small sample size, as only 12 patients had their $\mathrm{BH}$ planning CT scan acquired using the Abches monitoring system. In fact, as we dichotomized the data, we would have needed 12/12 (100\%) patients scanned with Abches to have a BH CT scan closer to EE than the EI 4DCT phase to see statistical significance (at a 5\% level) between the two groups. It would be interesting to conduct a similar study in a hospital where the Abches device is used on a daily basis to establish stronger conclusions.

Apart from sample size, this study has a few limitations. First, tumor motion was modeled as a linear movement. Motion non-linearity could have been assessed with deformable registration [12]. Secondly, we did not compare tumor motion on planning images to motion at the time of treatment [13]. Finally, planning 4DCT paths could have been compared to those estimated during treatment sessions with the CyberKnife (CK) Synchrony system.

Several hypotheses can be formulated to explain the relatively frequent presence of unnatural BH CT during treatment planning. Patients' anxiety might involuntarily induce respiratory muscle contraction during CT acquisition. Also, patients' ability to tolerate breath holding at EE state might be affected by their pulmonary function or comorbidity status. Thus, for selected cases, the use of a feedback device might not offer any benefit in treatment planning.

\section{Conclusions}

Tumor position during breath-hold CT may not accurately correspond to positions observed on free-breathing 4DCT. The use of a respiratory monitoring system did not significantly impact this issue. Hence, it is important to review a free-breathing 4DCT for all methods of motion management. For treatment with tumor tracking, an aberrant tumor position on a breath-hold scan could potentially impact dose to organs at risk. For patients treated with an internal target volume technique, treatment planning which includes tumor position during breath-hold will lead to an increase in irradiated lung.

\section{Appendices}


- $3 D$ BH CT: Performed close to expiration as seen on the respiratory trace with Bellows system. BH was well kept during acquisition. An extended FoV of $600 \mathrm{~mm}$ was used. The position of the tumor is aberrant compared with the natural free breathing trajectory. We recommend performing doses registration to ensure that constraints to OARs are respected.

- 4DCT: No audio coaching was used. Respiratory rate was stable in rate and amplitude. Respiratory phases $0 \%$ and $60 \%$ match inspiration and expiration, respectively.

- Markers: 4 markers are observed. In an order sup-inf, left-right, ant-post: markers 1 and 2 are located outside the tumor and markers 3 and 4 are located in the tumor.

- Registration: A marker-marker registration of the $0 \%$ and $60 \% 4 \mathrm{D}$ phases was performed on the $\mathrm{BH}$ scan. Measurements of the distance between pairs of markers were made. A registration error of less than $2 \mathrm{~mm}$ was observed for markers 3 and 4 vs $5 \mathrm{~mm}$ for markers 1 and 2 . Therefore, we recommend using markers 3 and 4 for tracking.

- Tumor deformation and rotation: Negligible deformation. No rotation of the tumor in the axial plane.

- Natural tumor motion: A tumor-tumor registration was performed from the $0 \%$ and $60 \%$ phases of the 4DCT. No significant hysteresis observed in the analysis.

\section{$8 \mathrm{~mm}$ sup-inf \\ $3 \mathrm{~mm}$ left-right \\ $4 \mathrm{~mm}$ ant-post}

\section{FIGURE 4: Sample Report : 4DCT and BH CT planning images for CK treatment using fiducials.}

\section{Additional Information}

\section{Disclosures}

Human subjects: Consent was obtained by all participants in this study. Animal subjects: All authors have confirmed that this study did not involve animal subjects or tissue. Conflicts of interest: In compliance with the ICMJE uniform disclosure form, all authors declare the following: Payment/services info: All authors have declared that no financial support was received from any organization for the submitted work. Financial relationships: Édith Filion Houda Bahig declare(s) a grant from Accuray Symposium on CK treatment for early lung cancer (TALK). This work is not related to the talk done during the CARO meeting in sept 2013. MariePierre Campeau declare(s) a grant from Accuray. Grant- study on SBRT in locally advanced pancreatic cancer. David Roberge declare(s) personal fees from Accuray. Lectures. Other relationships: All authors have declared that there are no other relationships or activities that could appear to have influenced the submitted work.

\section{Acknowledgements}

The authors thank Hideyuki Kawakami, president of APEX Medical inc, and Dr. Yoshiyasu Maehata from the University of Yamanashi for their research partnership and expertise. This 
work was presented in part at the 2013 SRS/SBRT Scientific Meeting, Carlsbad, California.

\section{References}

1. Onishi H, Kawakami H, Marino K, Kimiyama T, Kashiyama S, Sano N, Araki T, Ashizawa K, Matsuda S: A newly developed simple and accurate respiratory indicator relative to measurement of 2-point levels of abdominal and chest walls: for assurance of patient selfjudged breath holding techniques for irradiation of lung cancer with small internal margin. Proceedings of the American Society for Therapeutic Radiology and Oncology 47 th Annual Meeting. 2005, 63:S534. Accessed: November 27, 2014: http://dx.doi.org/10.1016/j.ijrobp.2005.07.902.

2. Bahig H, Campeau MP, Vu T, Doucet R, Béliveau Nadeau D, Fortin B, Roberge D, Lambert L, Carrier JF, Filion E: Predictive parameters of CyberKnife fiducial-less (XSight Lung) applicability for treatment of early non-small cell lung cancer: A single-center experience. Int J Radiat Oncol Biol Phys. 2013, 87:583-9. 10.1016/j.ijrobp.2013.06.2048

3. Shirato H, Onimaru R, Ishikawa M, Kaneko J, Takeshima T, Mochizuki K, Shimizu S, Umegaki K: Real-time 4-D radiotherapy for lung cancer . Cancer Sci. 2012, 103:1-6. 10.1111/j.13497006.2011.02114.x

4. Bengua G, Ishikawa M, Sutherland K, Horita K, Yamazaki R, Fujita K, Onimaru R, Katoh N, Inoue T, Onodera S, Shirato H: Evaluation of the effectiveness of the stereotactic body frame in reducing respiratory intrafractional organ motion using the real-time tumor-tracking radiotherapy system. Int J Radiat Oncol Biol Phys. 2010, 77:630-6.

10.1016/j.ijrobp.2009.08.060

5. Tarohda TI, Ishiguro M, Hasegawa K, Kohda Y, Onishi H, Aoki T, Takanaka T: The management of tumor motions in the stereotactic irradiation to lung cancer under the use of Abches to control active breathing. Med Phys. 2011, 38:4141-6.

6. Takamatsu S, Takanaka T, Kumano T, Mizuno E, Shibata S, Ohashi S, Kurata Y, Ueda S, Hori N, Shouji S, Noto K, Kojima H, Matsui O: Reproducibility of diaphragm position assessed with a voluntary breath-holding device. Jpn J Radiol. 2013, 31:357-63. 10.1007/s11604-013-0199-3

7. Lee HY, Chang JS, Lee IJ, Park K, Kim YB, Suh CO, Kim JW, Keum KC: The deep inspiration breath hold technique using Abches reduces cardiac dose in patients undergoing left-sided breast irradiation. Radiat Oncol J. 2013, 31:239-46. 10.3857/roj.2013.31.4.239

8. Wang L, Hayes S, Paskalev K, Jin L, Buyyounouski MK, Ma CC, Feigenberg S.: Dosimetric comparison of stereotactic body radiotherapy using 4D CT and multiphase CT images for treatment planning of lung cancer: Evaluation of the impact on daily dose coverage. Radiother Oncol. 2009, 91:314-24. 10.1016/j.radonc.2008.11.018

9. Liu HH, Balter P, Tutt T, Choi B, Zhang J, Wang C, Chi M, Luo D, Pan T, Hunjan S, Starkschall G, Rosen I, Prado K, Liao Z, Chang J, Komaki R, Cox JD, Mohan R, Dong L: Assessing respiration-induced tumor motion and internal target volume using four-dimensional computed tomography for radiotherapy of lung cancer. Int J Radiat Oncol Biol Phys. 2007, 68:531-40.

10. Plathow C, Ley S, Fink C, Puderbach M, Hosch W, Schmähl A, Debus J, Kauczor HU: Analysis of intrathoracic tumor mobility during whole breathing cycle by dynamic MRI. Int J Radiat Oncol Biol Phys. 2004, 59:952-9.

11. Ong C, Verbakel WF, Cuijpers JP, Slotman BJ, Senan S: Dosimetric impact of interplay effect on RapidArc lung stereotactic treatment delivery. Int J Radiat Oncol Biol Phys. 2011, 79:30511. 10.1016/j.ijrobp.2010.02.059

12. Boldea V, Sharp GC, Jiang SB, Sarrut D: 4D-CT lung motion estimation with deformable registration: Quantification of motion nonlinearity and hysteresis. Med Phys. 2008, 35:100818.

13. Seppenwoolde Y, Shirato H, Kitamura K, Shimizu S, van Herk M, Lebesque JV, Miyasaka K: Precise and real-time measurement of 3D tumor motion in lung due to breathing and heartbeat, measured during radiotherapy. Int J Radiat Oncol Biol Phys. 2002, 53:822-34. 